\title{
Transient Activity in the Human Calcarine Cortex During Visual-Mental Imagery: An Event-Related fMRI Study
}

\section{Citation}

Klein, Isabelle, Anne-Lise Paradis, Jean-Baptiste Poline, Stephen M. Kosslyn, and Denis Le Bihan. 2000. Transient activity in the human calcarine cortex during visual-mental imagery: An event-related fMRI study. Journal of Cognitive Neuroscience 12(Supplement 2): 15-23.

\section{Published Version}

doi:10.1162/089892900564037

\section{Permanent link}

http://nrs.harvard.edu/urn-3:HUL.InstRepos:3597234

\section{Terms of Use}

This article was downloaded from Harvard University's DASH repository, and is made available under the terms and conditions applicable to Other Posted Material, as set forth at http:// nrs.harvard.edu/urn-3:HUL.InstRepos:dash.current.terms-of-use\#LAA

\section{Share Your Story}

The Harvard community has made this article openly available.

Please share how this access benefits you. Submit a story.

\section{Accessibility}




\title{
Transient Activity in the Human Calcarine Cortex During Visual-Mental Imagery: An Event-Related fMRI Study
}

\author{
Isabelle Klein \\ SHFJ/DRM/DSV/CEA, Orsay, France \\ Anne-Lise Paradis \\ SHFJ/DRM/DSV/CEA, Orsay, France and LPPA-CNRS/College de France \\ Jean-Baptiste Poline \\ SHFJ/DRM/DSV/CEA, Orsay, France
}

\section{Stephen M. Kosslyn}

Harvard University and Massachusetts General Hospital

\section{Denis Le Bihan}

SHFJ/DRM/DSV/CEA, Orsay, France

\begin{abstract}
Although it is largely accepted that visual-mental imagery and perception draw on many of the same neural structures, the existence and nature of neural processing in the primary visual cortex (or area V1) during visual imagery remains controversial. We tested two general hypotheses: The first was that V1 is activated only when images with many details are formed and used, and the second was that V1 is activated whenever images are formed, even if they are not necessarily used to perform a task. We used event-related functional magnetic resonance imaging (ER-fMRI) to detect and char-
\end{abstract}

\section{INTRODUCTION}

Visual-mental imagery is a brain state that gives rise to visual experience on the basis of stored information, not current input from the eyes. Convergent results from psychophysical (Kosslyn, Ball, \& Reiser, 1978), neuroimaging (see Kosslyn, Thompson, \& Alpert, 1997; Roland \& Gulyas, 1995; Goldenberg, Steiner, Podreka, \& Deecke, 1992; Farah, Peronnet, Gonon, \& Giard, 1988) and studies of brain-damaged patients (Bisiach \& Luzzatti, 1978) have shown that mental imagery shares many of the functional properties and cortical structures used in visual perception.

However, a fundamental question that remains unresolved is whether visual imagery shares the "low-level" neural mechanisms required to visually perceive the external world. Although many neuroimaging studies acterize the activity in the calcarine sulcus (which contains the primary visual cortex) during single instances of mental imagery. The results revealed reproducible transient activity in this area whenever participants generated or evaluated a mental image. This transient activity was strongly enhanced when participants evaluated characteristics of objects, whether or not details actually needed to be extracted from the image to perform the task. These results show that visual imagery processing commonly involves the earliest stages of the visual system.

have provided support for the involvement of the primary visual cortex during visual imagery (e.g., Thompson \& Kosslyn, 2000; Chen et al., 1998; Kosslyn et al., 1993; Kosslyn, Thompson, Kim, \& Alpert, 1995; Kosslyn et al., 1999; Le Bihan et al., 1993), other studies have not found such activation (Mellet et al., 2000; Mellet, Tzourio, Denis, \& Mazoyer, 1998; D'Esposito et al., 1997; for reviews, see Mellet, Petit, Mazoyer, Denis, \& Tzourio, 1998; Roland \& Gulyas, 1994). It is possible that the observed activation in this region is simply "epiphenomenal," playing no role in information processing. However, performance in analogous imagery and perception tasks was impaired when neural activity in the medial-occipital cortex was temporarily disrupted by repetitive transcranial magnetic stimulation (rTMS; Kosslyn et al., 1999). Such results suggest therefore that 
early visual areas do in fact play a causal role during mental imagery. Thus, we are left with the mystery of why some studies have found activation in these areas, but others have not.

Two classes of accounts for these disparate results can be formulated. First, the nature of the imagery tasks varied in the different experiments, and these differences may be crucial (see Thompson \& Kosslyn, 2000; Kosslyn \& Ochsner, 1994; Sakai \& Miyashita, 1994). In particular, the primary visual cortex was activated during most imagery tasks that required very detailed images (Kosslyn et al., 1993, Kosslyn et al., 1995, Kosslyn et al., 1999) or short-term visual recall (e.g., Chen et al., 1998; Le Bihan et al., 1993). It is possible that V1, with its highspatial resolution, is necessary only when high levels of detail must be added to the imaged object in order to perform the task (note: given the results of Mellet et al., 2000 , it is unlikely that the requirement to use high resolution per se is crucial; rather, it may be the requirement to add details that rely on high resolution that is critical). Second, differences in the sensitivity of the methods may lie at the heart of the disparate results; studies that failed to detect activation in early visual areas generally relied on the averaging over multiple participants and trials. It is possible either that some previous tasks did not require enough imagery so that the accompanying activation could be detected, or that the methods simply were not sensitive enough to detect activation in this region.

In an effort to distinguish between these alternatives, we used the high-temporal resolution and sensitivity of event-related functional magnetic resonance imaging (ER-fMRI). This technique is significantly more sensitive than previous methods, enabling measures of cerebral activity in relation to a cognitive task (e.g., see Konishi et al., 1998; Richter, Ugurbil, Georgopoulous, \& Kim, 1997; Buckner et al., 1996). At present, most functional neuroimaging studies of mental imagery have relied upon the classical "block-design" paradigm, where participants must generate mental images for relatively long periods (e.g., $1 \mathrm{~min}$ ).

In the present study, we measured cerebral activity along the calcarine sulcus (which contains the primary visual cortex) when participants, with eyes closed and in complete darkness, were asked to perform individual imagery trials. There were two events on each trial: first, participants heard the name of an animal and formed a visual image of it (we will refer to this first event as E1); and second, 14 sec later, they heard a possible characteristic of the animal and decided, as quickly and accurately as possible, whether the animal did in fact have that characteristic (half the time it did, and half the
Figure 1. Task design. A single imagery trial is represented for each condition. During concrete trials, participants were told first to visualize a named animal (E1), then to evaluate whether that animal has a named characteristic only after having visualized relevant attributes (E2). During abstract imagery trials, participants were first asked to visualize the named animal (E1), and then (E2) to evaluate the abstract characteristic as quickly and accurately as possible. In this condition, characteristics were not directly visible, but could be inferred indirectly from behaviors; participants were told that visible information was not necessary to perform the task. Each type of trial was presented in a group in sequences lasting 280 sec. Before starting each sequence, participants were informed of the "concrete" or "abstract" nature of the subsequent characteristics and were asked to close their eyes.

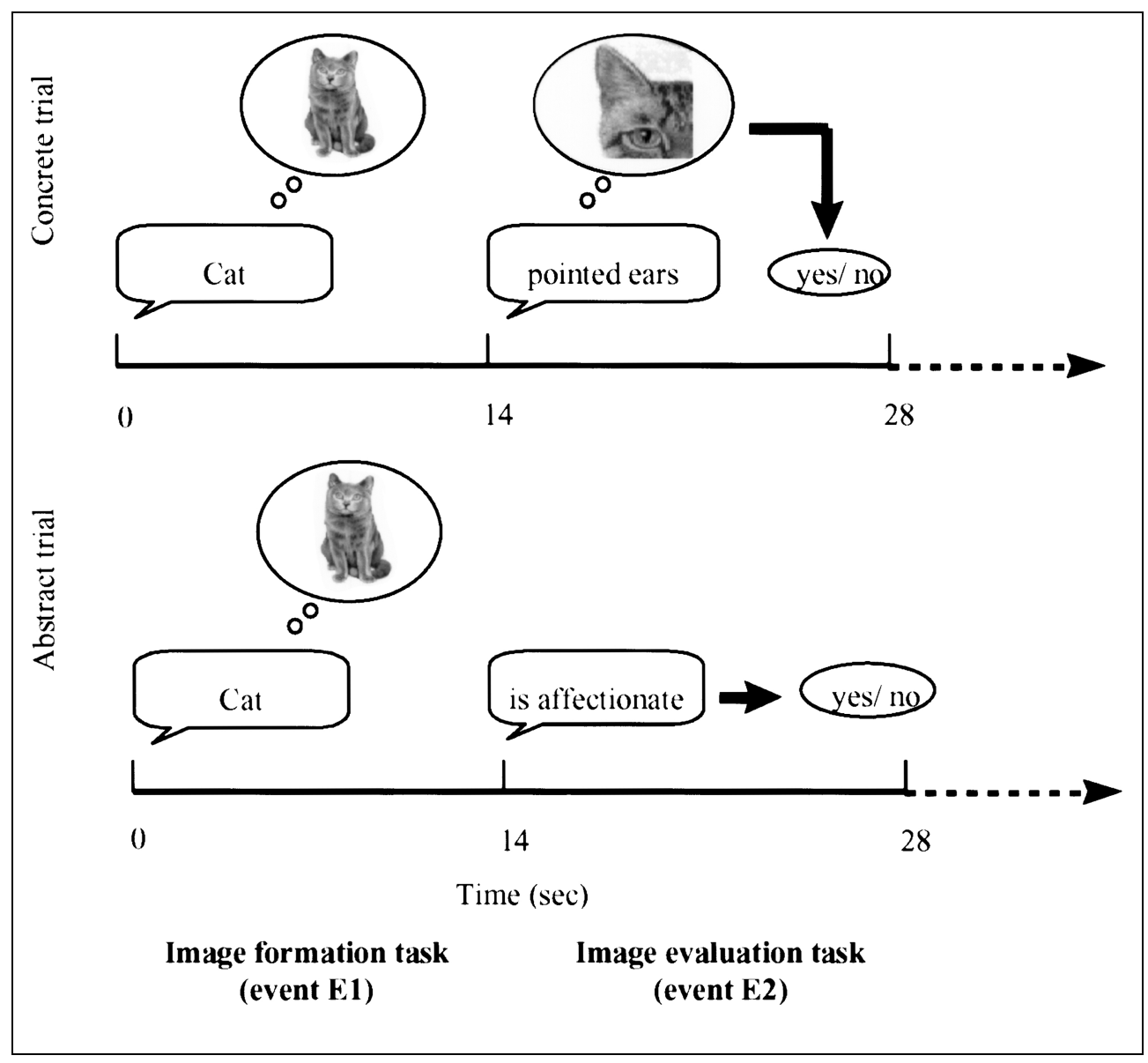


time it did not; we will refer to this second event as E2). Thus, we had a measure of activation when participants formed an image and another when they evaluated a characteristic of the object.

In addition, in separate sets of trials, we probed two types of characteristics: In one, the "concrete characteristic" trials, the characteristics were visible details of the body. We asked the participants to use their images of the animals to evaluate these characteristics, e.g., they first may have heard "cat," and then "pointed ears." In this case, the participant was to decide whether cats have pointed ears by "inspecting" the image. In the other set, the "abstract characteristic" trials, participants evaluated character- istics that were not descriptions of body parts, but instead named abstract characteristics, e.g., "cat" and "is affectionate." To perform this task, participants were told to make their judgments as quickly and accurately as possible without necessarily "looking" at the image (Figure 1). If the amount of detail necessary to perform the task is crucial, then we should find activation in the calcarine cortex when participants form images in anticipation of using them to answer questions about concrete details (during E1). Moreover, we should find more activation when they use the image to "inspect" the details (during E2). By the same token, if this hypothesis is correct, then we should not find much activation, if any,
Figure 2. Time course of imagery-related BOLD responses in the calcarine cortex. (a) Location of the voxels that had maximal $t$ values $(p<.001)$ along the calcarine sulcus (white arrows on the sagittal anatomical section) during the "evaluation" task (E2) in one representative participant. (b) The individual time course of imagery-related signal increases from the maximal activated voxel (Talairach coordinates: $-8-928$ ) detected in the calcarine cortex during the performance of ten 28 -sec concrete and abstract imagery trials are shown on the two graphs (green and red curves, respectively, with fitted estimation in gray lines). In each condition, a BOLD response could be monitored during each single event. (c) Averaged BOLD responses of the calcarine maximal activated clusters during 60 trials of concrete (green curve) and abstract trials (red curve). The averaged BOLD response in relation to each type of event (E1 and E2) is represented during one 28-sec imagery trial. These curves illustrate that the response in the calcarine cortex increased with high reproducibility when participants evaluated either concrete or abstract characteristics.

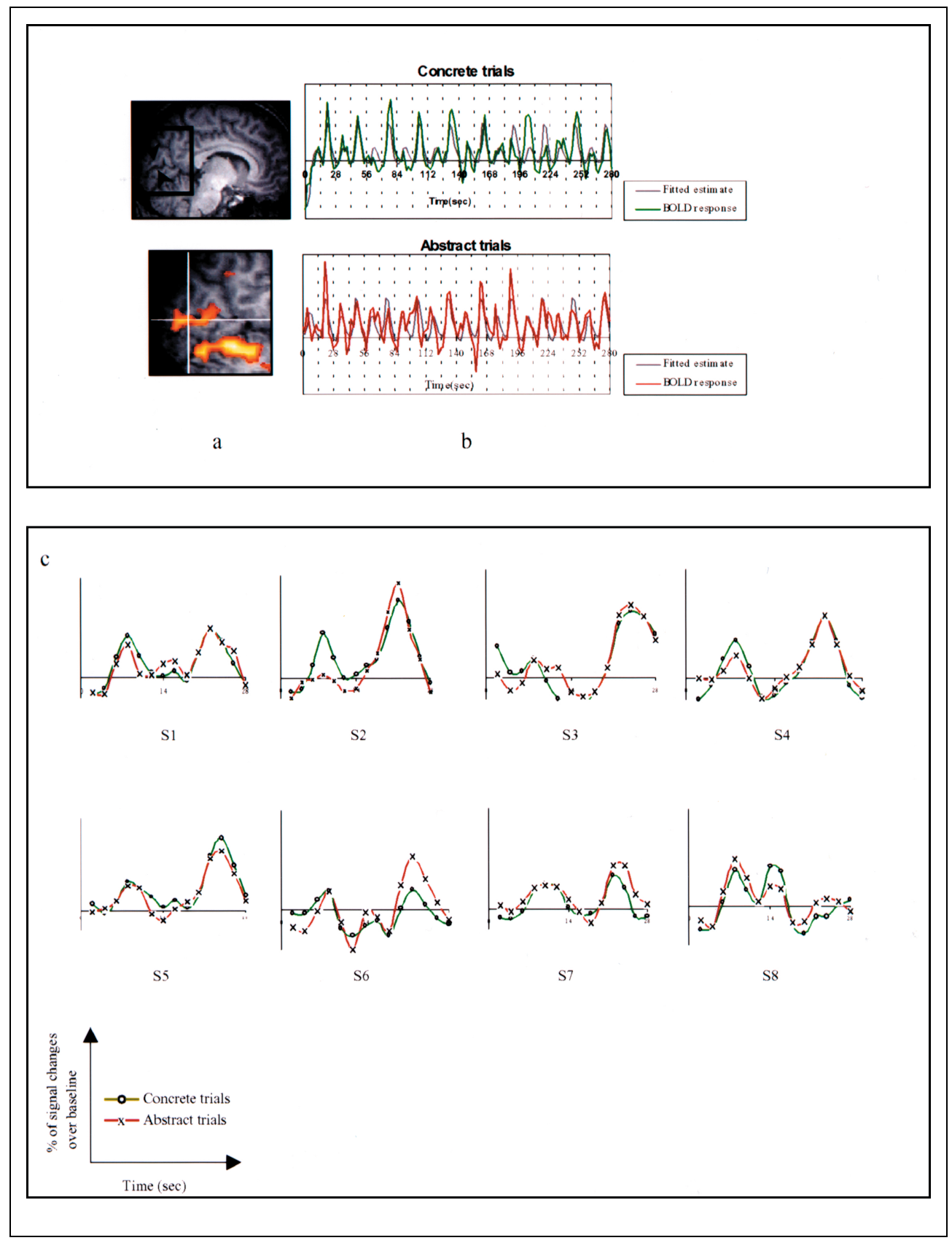


Table 1. Activation in the Calcarine Cortex During Visual Imagery

\begin{tabular}{|c|c|c|c|c|}
\hline \multirow[b]{2}{*}{ Subjects } & \multicolumn{2}{|c|}{$\begin{array}{l}\text { Image formation } \\
\text { task (E1) }\end{array}$} & \multicolumn{2}{|c|}{$\begin{array}{l}\text { Image evaluation } \\
\text { task (E2) }\end{array}$} \\
\hline & $\begin{array}{c}\mathrm{Nb} \\
\text { voxels }\end{array}$ & $\begin{array}{c}\text { Peak } \\
\text { z value }\end{array}$ & $\begin{array}{c}\mathrm{Nb} \\
\text { voxels }\end{array}$ & $\begin{array}{c}\text { Peak } \\
\text { z value }\end{array}$ \\
\hline \multicolumn{5}{|c|}{ Concrete imagery } \\
\hline No. 1 & $12(4)$ & 4.7 & $28(4)$ & 6.4 \\
\hline No. 2 & $4(1)$ & 3.9 & $56(2)$ & 7.3 \\
\hline No. 3 & $3(1)$ & 3.7 & $55(1)$ & 7.8 \\
\hline No. 4 & $6(3)$ & 5 & $51(4)$ & 7.2 \\
\hline No. 5 & $10(3)$ & 4.2 & $64(4)$ & 7.8 \\
\hline No. 6 & $3(1)$ & 3.4 & $4(1)$ & 3.6 \\
\hline No. 7 & $20(4)$ & 6.4 & $41(3)$ & 7.3 \\
\hline No. 8 & $3(1)$ & 4.3 & 0 & 0 \\
\hline \multicolumn{5}{|c|}{ Abstract imagery } \\
\hline No. 1 & $6(1)$ & 4.1 & $54(1)$ & 7.4 \\
\hline No. 2 & $2(1)$ & 3.1 & $54(2)$ & 7.4 \\
\hline No. 3 & $3(1)$ & 3.8 & $59(1)$ & 7.9 \\
\hline No. 4 & $12(2)$ & 7.3 & $59(4)$ & 7 \\
\hline No. 5 & $9(2)$ & 4.5 & $57(3)$ & 7.6 \\
\hline No. 6 & $3(1)$ & 3.5 & $7(2)$ & 5.4 \\
\hline No. 7 & $29(2)$ & 7 & $55(2)$ & 7.6 \\
\hline No. 8 & $8(1)$ & 4.6 & 0 & 0 \\
\hline
\end{tabular}

For all eight participants, the extent of calcarine activation is reported in each condition by the number of voxels exceeding the significance level of $p=.001$, with the number of clusters identified within each VOI in parentheses. The peak $z$ value is also given $(z>3.09$ corresponding to $p<.001$ ).

when participants form an image in anticipation of evaluating an abstract characteristic (during E1), and none when they actually evaluate the characteristic (during E2).

\section{RESULTS}

Performance data showed that on average, participants required more time to evaluate the concrete characteristics than the abstract ones, with mean response times of 4,479 and 3,503 msec (paired $t$ test, $t(7)=2.3, p=$ .05 ), and had corresponding error rates of $23 \%$ and $18 \%$. This difference could be taken as evidence that the participants processed the two types of trials differently, but they may in fact merely indicate that the concrete characteristics were less familiar or, otherwise, more difficult to evaluate.

Significant activation was detected in the calcarine sulcus in all imagery conditions when participants formed images (E1), and when they evaluated the characteristics (E2). This calcarine activation was detected in both concrete and abstract conditions.

\section{Time-Course Analysis}

The time course of this calcarine activity was then examined, which reproducibly showed that a transient increase in BOLD response could be observed in the calcarine cortex for each individual imagery event over the course of both concrete and abstract sessions (Figure 2b). In order to consider the waveform and duration of the hemodynamic response, we computed for each participant the averaged signal for each event on each trial. The time course of activation was determined by averaging the time courses of all activated voxels within the maximal calcarine-activated cluster. The procedure used to define calcarine-active clusters is detailed in the Methods section. This averaging was performed separately for the concrete and abstract conditions. As shown in Figure 2c, the BOLD response reproducibly evolved over a $12-14$-sec period, showing a delayed positive deflection about $2 \mathrm{sec}$ after the

Table 2. The Effect of Concrete and Abstract Conditions on Calcarine Activation is Reported in Terms of Peak $z$ Values, with the Number of Activated Voxels in Parentheses

\begin{tabular}{|c|c|c|c|c|c|c|}
\hline Subjects & E1Conc. vs. E1Abs. & E2Conc. vs. E2Abs. & Conc. vs. Abs. & E1Abs. vs. E1Conc. & E2Abs. vs. E2Conc. & Abs. vs. Conc. \\
\hline No. 1 & - & - & - & - & - & - \\
\hline No. 2 & $3.5(3 v)$ & - & - & - & - & - \\
\hline No. 3 & - & - & - & - & - & - \\
\hline No. 4 & $3.47(3 v)$ & - & - & - & - & - \\
\hline No. 5 & - & - & $4.5(3 v)$ & - & - & - \\
\hline No. 6 & - & - & - & - & $3.8(4 v)$ & - \\
\hline No. 7 & - & - & - & - & $4.7(4 v)$ & $3.8(4 v)$ \\
\hline No. 8 & - & - & - & - & - & - \\
\hline
\end{tabular}


Figure 3. Calcarine activation maps. Top row: Locations of maximal activated voxels in the calcarine sulcus (centered on white cursor) were identified for each participant on 3-D anatomical sections. Bottom row: Individual corresponding coronal sections of activation maps of the contrast "evaluation" versus "formation" tasks averaged over concrete and abstract imagery trials in seven out of eight participants (a). (b) This figure illustrates in participant no. 8 the resulting activation map of the calcarine cortex for the contrast "formation" versus "evaluation" (E1 vs. E2) averaged over concrete and abstract trials. The left hemisphere appears on the right in all images.

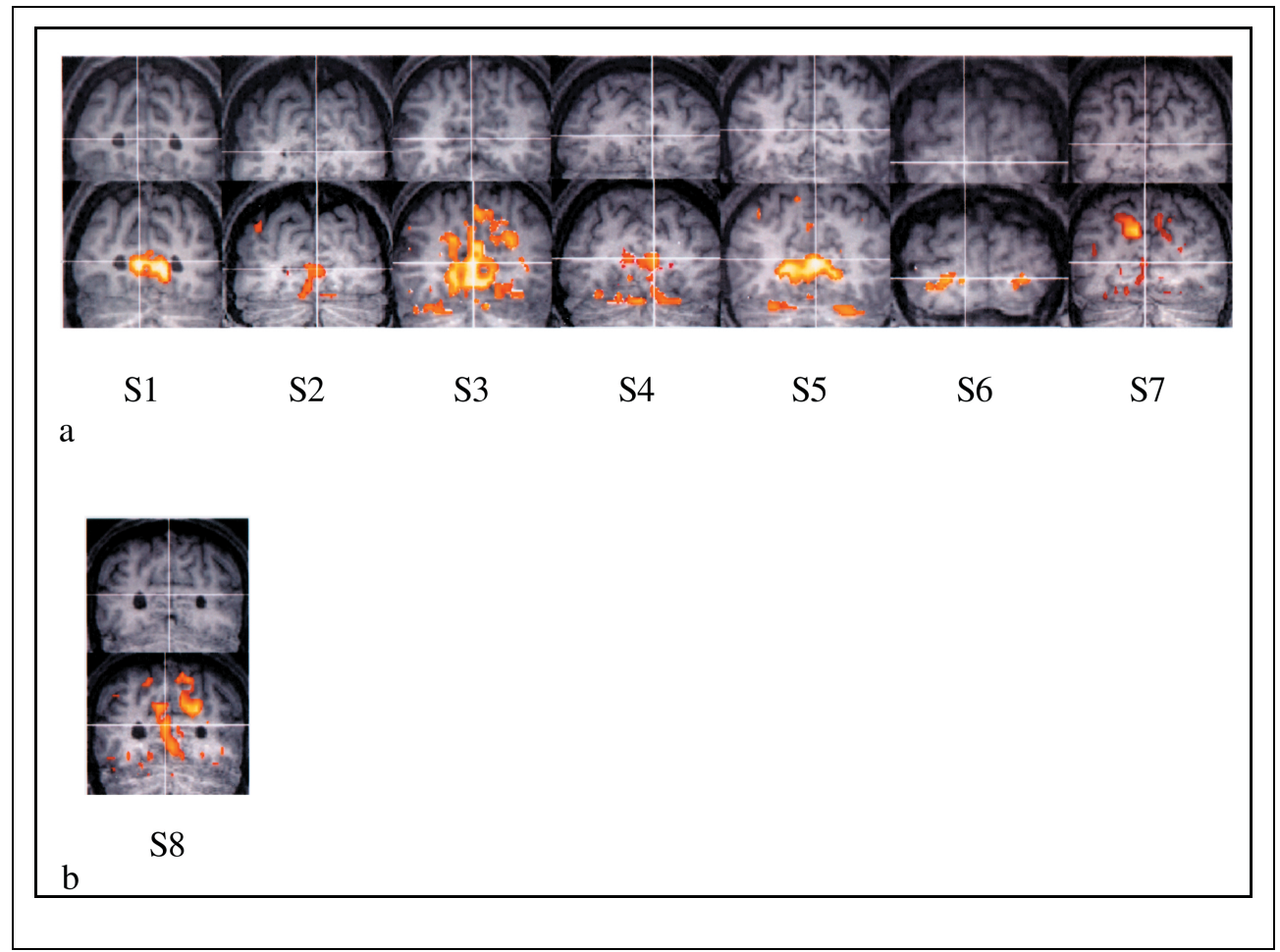

verbal cue was presented and reaching a peak at around 6-8 sec.

\section{Extent Analysis}

We then analyzed the pattern of activation in the calcarine cortex. We measured for each participant and condition the number of voxels of clusters that had significant activation in the calcarine sulcus (see Methods). As summarized in Table 1, we observed that the extent of activation in the calcarine cortex varied a large amount across participants. As is evident, the extent of activation in the calcarine cortex did not differ for the two imagery conditions across participants (paired $t$ test, $t(7)=.3, p=.76)$.

\section{Amplitude Analysis}

By comparing the amplitude of the response in the calcarine cortex as obtained for each condition during each type of trial (i.e., E1 and E2), we could not detect any differential effect of the imagery condition on the amplitude of response in the calcarine cortex (paired $t$ test, $t(7)=.5, p=.63)$.

Hence, to analyze more closely the effect of the concrete versus the abstract imagery condition on calcarine

Table 3. The Effect of the Task on Calcarine Activation is Reported in Terms of Peak $z$ Values and the Number of Significantly Activated Voxels for Each Contrast in Parentheses

\begin{tabular}{|c|c|c|c|c|c|c|}
\hline Subjects & E1Conc. vs. E2Conc. & E1Abs. vs. E2Abs. & E1 vs. E2 & E2Conc. vs. E1Conc. & E2Abs. vs. E1Abs. & E2 vs. E1 \\
\hline No. 1 & - & - & - & $4.46(9 v)$ & $6.5(31 v)$ & $5.59(14 \mathrm{v})$ \\
\hline No. 2 & - & - & - & $5.43(17 v)$ & $7.86(55 v)$ & $8.24(65 v)$ \\
\hline No. 3 & - & - & - & $8.07(66 v)$ & $7.78(59 v)$ & $8.5(68 v)$ \\
\hline No. 4 & - & - & - & $4.9(10 v)$ & $6.05(54 \mathrm{v})$ & $7.04(63 v)$ \\
\hline No. 5 & - & - & - & $7.5(56 \mathrm{v})$ & $7.34(59 v)$ & $8.2(69 v)$ \\
\hline No. 6 & - & - & - & - & $7.48(21 v)$ & $7.4(17 \mathrm{v})$ \\
\hline No. 7 & - & - & - & - & $4.66(13 v)$ & $4.15(12 \mathrm{v})$ \\
\hline No. 8 & $5.04(10 v)$ & $4.8(8 v)$ & $6.2(13 v)$ & - & - & - \\
\hline
\end{tabular}


activation, we computed for each participant the activation maps of the direct comparison between the response to concrete imagery with the response to abstract imagery (and vice-versa). As reported in Table 2, we found that only two participants (no. 2 and no. 4) had more activation in the calcarine cortex when forming an image in anticipation of having to evaluate a concrete characteristic than when forming an image in preparation for the abstract evaluation (in "E1Conc. vs. E1Abs."). When we contrasted the response elicited by the evaluation of concrete characteristics to the response to the evaluation of abstract characteristics ("E2Conc. vs. E2Abs."), we did not detect significant activation in the calcarine cortex (with $p=.001$ ). However, two participants (no. 6 and no. 7) showed the reverse effect, having more activation on trials with abstract characteristics ("E2Abs. vs. E2Conc."). By pooling E1 and E2 data for each participant, we found only one (no. 5) who had more activation in the calcarine cortex during concrete imagery ("Conc. vs. Abs.") and one (no. 7) who had more activation for the reverse contrast ("Abs. vs. Conc."). In short, overall, we did not find any significant effect of the concrete versus abstract imagery condition on calcarine activation.

We then compared the image formation task (E1) and the evaluation task (E2). The contrast "evaluation" versus "formation" ("E2 vs. E1") showed that seven of eight participants had more activity in the calcarine cortex when they evaluated characteristics than when they formed the images initially (Figure 3a), as presented in Table 3 . This enhancement of activation was found when combining together the responses to concrete and abstract trials. When we examined the reverse contrast, only one participant (no. 8) showed stronger activation along the calcarine sulcus during E1 than during E2 (Table 3 and Figure 3b).

\section{Correlation Analysis Between Response Times and Calcarine Activation}

We then asked whether the amount of activation in the calcarine cortex correlated with the response times during the evaluation task. The Pearson correlation was calculated between the averaged peak response of the maximal activated calcarine cluster and the response time recorded for each participant on each trial. Only response times on trials in which participants responded correctly were included. The analysis was performed separately for concrete and abstract characteristics. To select activated clusters, we used the same procedure used for BOLD response analysis. For peak response, we considered the maximal signal value at each voxel, then averaged these peak values across all voxels. Across all participants, we found that the response in the calcarine cortex to visual imagery was significantly negatively correlated with the evaluation times during both concrete and abstract conditions (with $r(182)=-.20, p=$ .001 , and $r(193)=-.16, p=.01$, respectively). We did not find any correlation between response times and the response in the calcarine cortex when participants responded incorrectly (with $r(56)=-.04, p=.77$ and $r(45)=-.08, p=.56$, respectively, for concrete and abstract imagery).

\section{DISCUSSION}

The results were clearcut: the calcarine cortex was activated when participants formed images, and was activated to the same degree whether or not high detail was actually necessary to perform the task. Indeed, to our surprise, this region was activated even when participants did not actually need to use the image to evaluate an abstract characteristic - although in these cases, the image was apparently present, e.g., when deciding whether a cat is affectionate, participants sometimes reported visualizing a cat and "seeing" it rub against someone's shin. One possible explanation is that the "abstract" condition was not as abstract as we hoped, because the task involved evaluating characteristics of concrete "objects" (animals) that can, in fact, be visualized. However, only two participants (no. 5 and no. 7) said that they actually "looked at" the image to help them evaluate the abstract characteristics. Thus, simply forming an image seems to be enough to activate the calcarine cortex.

How, then, can we explain previous failures to find this effect? To our knowledge, this study is the first to report transient signal changes along the calcarine sulcus when participants, with eyes closed and in complete darkness, form images and evaluate imaged objects. It is unlikely that responses measured in the calcarine cortex during visual imagery are related to hearing the auditory cues because neuroimaging studies of the modulation of primary sensory cortices have reproducibly shown that selective attention to auditory or visual stimuli activates modality-dependent cortical areas (Bushara et al., 1999; Kawashima et al., 1999; Woodruff et al., 1996).

This technique, thus, appears to be significantly more sensitive than many of those used previously. Not only could neural activity be measured for each averaged trial type, but also, a single BOLD response could be monitored in this region during single visual imagery events, indicating that in the absence of retinal input, the formation of a single visual-mental image can induce a response in the earliest stages of the visual system. These ER-fMRI data also highlight the importance of individual variability, even within a single imagery session; such variability may have influenced previous results, particularly when data were averaged across long imagery periods (such as with block paradigm fMRI or positron emission tomography [PET] studies).

Although our data strongly suggest that methodological issues may be responsible for the previous "negative" results of V1 activation, our results indicate that selective neural mechanisms may modulate the activity of primary visual cortex during visual imagery. Indeed, in 
seven out of eight participants, the amount of activation in the calcarine cortex was robustly larger (in terms of size of activation and in terms of magnitude of response) when participants evaluated either a concrete or an abstract characteristic (E2), than when they simply formed an image (E1). These mechanisms presumably are related to those used in attention; in fact, researchers have found that attention can modulate activation in V1 during visual perception (Brefczynski \& De Yoe, 1999; Martinez et al., 1999; Somers, Dale, Seiffert, \& Tootel, 1999). Because successive events were separated in time by $14 \mathrm{sec}$, the enhanced activity observed in E2 cannot reflect the summation of BOLD responses elicited by preceding imagery events (Fransson, Krüger, Merboldt, \& Frahm, 1998).

Furthermore, our results indicate that less activation in the calcarine cortex was accompanied by longer evaluation times. This finding is consistent with the results of Kosslyn, Thompson, Kim, Albert, and Rauch (1996), who found that participants with less regional cerebral blood flow (measured using PET) in V1 required more time to evaluate imaged letters for particular characteristics (such as the presence of a curved line). This finding is consistent with the general conclusion that task difficulty is not crucial for activation of V1 during visual-mental imagery; the strength of the activation in primary visual cortex during imagery does not increase with more difficult tasks. However, the difference between E1 and E2 indicates clearly that properties of the task are in fact important.

Because our design required participants to perform successive tasks that were linked to the same object in both the concrete and abstract conditions, it can be asked whether short-term visual recall of the image formed in E1 may have created "lingering activation" that boosted activation during E2. Although imagery was not necessary to evaluate abstract characteristics, apparently having just formed an image (in E1), "primed" the participants so that they formed images even when evaluating these characteristics. This would be consistent with earlier results indicating that neurons in V1 may retain information over time (Ishaï \& Sagi, 1995). It is possible that such a "low-level" memory system during visual imagery subserves spatio-temporal associations across a transient time-window of a few seconds. To test this idea, one needs only to conduct a study like that reported here but in which E1 is omitted; if the present results are a consequence of having formed an image prior to evaluating that object, then when E1 is eliminated, activation in V1 should not occur when participants evaluate abstract characteristics.

Although understanding the precise nature of neural mechanisms that causally link activity in V1 to visual imagery requires further investigation, the present finding of transient and enhanced neural activity in the calcarine cortex provides strong evidence that visual-mental imagery recruits the earliest stages of the visual system.

\section{METHODS}

\section{Participants, Task, and Stimuli}

Eight participants (four male and four female, ages 20 to 25 years) volunteered to take part in the study. All participants gave their informed written consent. The study was approved by an Institutional Ethic Committee (\#95-23).

Participants received six replicates of 10 imagery trials. Each imagery trial began with a 2-sec auditory presentation of the name of an animal (E1) followed 14 sec later by a 2 -sec auditory presentation of a possible characteristic of the animal (E2). The six replicates were grouped into three experimental sessions. Each session consisted of two runs of either a concrete or abstract imagery condition. Participants reported their judgments for each characteristic by pressing a "yes" or "no" response key, with one key placed in each hand.

At the beginning of each replicate, participants were visually instructed about the nature of the subsequent set of characteristics. Thus, when generating the images in E1, they knew whether they would subsequently be asked to evaluate a concrete or abstract characteristic. This instruction was followed by a 14-sec rest period, where participants were told to close their eyes and relax.

A total of 60 names of animals were selected from a common French dictionary with the constraint that they were frequently used and had between two and three syllables. We formulated one concrete and one abstract characteristic for each animal. Concrete characteristics were easily visible parts, with each characteristic being described by between two and four syllables (such as "long ears"). Abstract characteristics were instead a behavioral tendency (such as "quite brave"), and these descriptors were between three and five syllables. Half the characteristics of each type did describe the animal, and half did not. Prior to the actual experiment, the characteristics were evaluated by 10 volunteers, who did not participate in the fMRI experiment (error rates: 13\% for abstract characteristics, 25\% for concrete characteristics).

Each animal appeared equally often with each type of characteristic. Each animal appeared only once with each type of characteristic, and did not appear more than once per run. The order of concrete and abstract conditions was counterbalanced across participants. Animals and characteristics were presented in a random order, except that no more than three "yes" or "no" characteristics appeared in succession. Participants received two practice runs before the actual experiment.

\section{Imaging}

Eighteen contiguous, axial, 5-mm-thick slices were acquired in three sessions of 308 scans each (repeat time, 2 sec) covering the whole occipital cortex. Six seconds of "dummy" gradient and radiofrequency (RF) pulses pre- 
ceded the actual data acquisition; fMRI data were acquired with a T2*-weighted gradient EPI sequence $(\mathrm{TE}=$ $40 \mathrm{msec}$, flip angle $90^{\circ}, 64 \times 80$ pixels in a $24-\times 30-\mathrm{cm}$ field-of-view) on a $3 \mathrm{~T}$ whole-body MRI system (Brucker, Karlsruhe, Germany). A standard RF head coil was used.

To help the participants hear the verbal instructions more easily, we employed a silent "non-encoding" sequence by removing the gradient pulses used along the readout axis of the EPI sequence during the first brain volume acquisition of each epoch (Van de Moortele, Le Clec'H, Dehaene, \& Le Bihan, 1998). Auditory and visual stimuli were delivered through a PC computer synchronized with fMRI acquisitions, using "EXPE5" software (Pallier, Dupoux, \& Jeannin, 1997). "Yes" or "no" responses were recorded on-line, where response time was measured from the time of stimulus onset to the key response.

A T1-weighted 3-D Inversion-Recovery Fast Gradient Echo sequence for anatomical identification of activated foci $(128 \times 128 \times 256$ matrix, $1.5 \times 1.5 \times 1 \mathrm{~mm}$ resolution) was acquired following functional imaging. All experimental sessions occurred in complete darkness.

\section{Data Analysis}

Functional data from individual ER-fMRI series were first corrected for different slice acquisition time (using IDL software) and then, using Statistical Parametric Mapping (SPM 96; Friston, Jezzard, \& Turner, 1994), they were corrected for participant motion. Data were then transformed into the stereotaxic atlas space (Talairach \& Tournoux, 1988) through a linear transform that was calculated by fitting the Montreal Neurological Institute (MNI) template. A spatial smoothing of $5 \mathrm{~mm}$ was applied to functional images using a gaussian spatial filter. Data were also highpass-filtered using a cut-off period of $60 \mathrm{sec}$ (about twice the period of the paradigm) to correct for temporal drift.

Statistical analyses were performed on an individual basis. The fMRI time course in each voxel during visual imagery was analyzed using multiple linear regression with regressors related to the four different imagery conditions: (1) a response to E1 in the "concrete" condition; (2) a response to E2 in the "concrete" condition; (3) a response to E1 in the "abstract" condition; (4) a response to E2 in the "abstract" condition. For all conditions, we used the same model of hemodynamic response, assuming that each event would evoke a BOLD response immediately after the 2 -sec verbal cue.

Other effects were also modeled according to the experimental conditions (visual, auditory instructions, and motor responses). In particular, response times recorded on each trial during imaging were also taken into account for the model of the motor response. The effects of interest were orthogonalized, which permitted responses to overlapping auditory events to be separated.

We computed statistical activation maps of: (1) the response to each event, corresponding to the amplitude of response normalized to the residual variance; (2) the contrasts between the conditions ("concrete" and "abstract" and vice-versa); (3) the contrasts between E1 versus E1, and vice-versa. These maps were thresholded using a voxel-wise significance level of $p=.001$. Because this analysis was directed specifically at the primary visual cortex, we did not correct for multiple comparisons (extent threshold: $p=.5$ ).

The location of the primary visual cortex was defined anatomically by identifying active voxels lying in the calcarine sulcus. Because there is an important interindividual variability in the location of the calcarine sulcus, this region was individually identified on 3-D anatomical sections for each participant. Activated clusters within the calcarine cortex were extracted by outlining a Volume of Interest (VOI) from the center of the calcarine sulcus, which was determined for each participant. We considered only voxels that were enclosed in the calcarine cortex and that exceeded the significance threshold of $p=.001$. For anatomical location, we chose as criterion that the Talairach coordinates of voxels should correspond to the Talairach coordinates of the calcarine sulcus. A simple visual-perceptual task, was also administered at the beginning of each imagery run to provide a coarse localization of occipital-visual areas. This task consisted in presenting a visual instruction that informed of the "concrete" or "abstract" nature of the subsequent characteristics. For the computation of averaged time courses, we chose the maximal activated cluster at each VOI. To estimate the effect of the imagery condition in terms of extent across participants, we counted up all the voxels of clusters that showed a significant effect in the calcarine cortex accounted for by the concrete imagery regressor, on the one hand, and by the abstract regressor, on the other hand, and then performed a paired $t$ test. For amplitude, we used the same approach to select active calcarine voxels, but took the mean $t$ value of all voxels showing an experimental effect in each condition and perform the $t$ test across participants.

\section{Acknowledgments}

We gratefully acknowledge S. Dehaene and M. Denis for their helpful comments. S. Kosslyn was supported in part by $\mathrm{NIH}$ grants from the Fogarty Institute and NIMH.

Reprint requests should to be sent to D. Le Bihan, Service Hospitalier Frédéric Joliot, 4 place du General Leclerc, Orsay 91401 France.

The data reported in this experiment have been deposited in National fMRI Data Center (http://www.fmridc.org). The accession number is 2-2000-11127. 


\section{REFERENCES}

Bisiach, E., \& Luzzatti, C. (1978). Unilateral neglect of representational space. Cortex, 14,129-133.

Brefczynski, J. A., \& De Yoe, E. A. (1999). A physiological correlate of the "spotlight" of the visual attention. Nature Neuroscience, 2, 370-374.

Buckner, R. L., Bandettini, P. A., O'Craven, K. M., Savoy, R. L., Petersen, S. E., Raichle, M. E., \& Rosen, B. R. (1996). Detection of cortical activation during averaged single trials of a cognitive task using functional magnetic resonance imaging. Proceedings of the National Academv of Sciences. U.S.A. 93, 14878-14883.

Bushara, K. O., Weeks, R. A., Ishii, K., Catalan, M. J., Tian B., Rauschecker, J. P., \& Hallett, M. (1999). Modality-specific frontal and parietal areas for auditory and visual spatial localization in humans. Nature Neuroscience. 2, 759-766.

Chen, W., Toshinori K., Xiao-Hong, Z., Ogawa, S., Tank, D. W., \& Ugurbil, K. (1998). Human primary visual cortex and lateral geniculate nucleus activation during visual imagery. NeuroReport. 9, 3669-3674.

D'Esposito, M., Detre, J. A., Aguirre, G. K., Stallcup, M., Alsop, D. C., Tippet, L. J., \& Farah, M. J. (1997). A functional MRI study of mental image generation. Neuropsvchologia. 35, 725-730.

Farah, M. J., Peronnet, F., Gonon, M. A., \& Giard, M. H. (1988). Electrophysiological evidence for a shared representational medium for visual images and visual percepts. Iournal of Experimental Psychology General. 117, 248-257.

Fransson, P., Krüger, G., Merboldt, K. D., \& Frahm, J. (1998). Physiologic aspects of event related paradigms in magnetic resonance functional neuroimaging. NeuroReport. 9, 2001-2005.

Friston, K. J., Jezzard, P., \& Turner, R. (1994). Analysis of functional MRI time series. Human Brain Mapping. 1, 153-171.

Goldenberg, G., Steiner, M., Podreka, I., \& Deecke, L. (1992). Regional cerebral blood flow patterns related to verification of high and low imagery sentences. Neuropsychologia. 30, 1081-1092.

Ishaï, A., \& Sagi, D. (1995). Common mechanisms of visual imagery and perception. Science. 268, 1772-1774.

Kawashima, R., Imaizumi, S., Mori, K., Okada, K., Goto, R., Kiritani, S., Ogawa, A., \& Fukuda, H. (1999). Selective visual and auditory attention toward utterances-a PET study. Neuroimage, 10, 209-215.

Konishi, S., Nakajima, K., Idai, I., Kameyama, M., Nakahara, K., Sekihara, K., \& Miyashita, Y. (1998). Transient activation of inferior prefrontal cortex during cognitive set shifting. Nature Neuroscience, 1, 80-84.

Kosslyn, S. M., Alpert, N. M., Thompson, W. L., Maljkovic, V., Weise, S. B., Chabris, C. F., Hamilton, S. E., Rauch, S. L., \& Buonanno, F. S. (1993). Visual mental imagery activates topographically organized visual cortex: PET investigations. Journal of Cognitive Neuroscience, 5, 263-287.

Kosslyn, S. M., Ball, T. M., \& Reiser, B. J. (1978). Visual images preserve metric spatial information: Evidence from studies of image scanning. Journal of Experimental Psychology: Human Perception and Performance, 4, 47-60.

Kosslyn, S. M., \& Ochsner, K. N. (1994). In search of occipital activation during visual mental imagery. Trends in Neurosciences, 17, 290-292.

Kosslyn, S. M., Pascual-Leone, A., Felician, O., Camposano, S., Keenan, J. P., Thompson, W. L., Ganis, G., Sukel, K. E., \& Alpert, N. M. (1999). The role of area 17 in visual imagery: Convergent evidence from PET and rTMS. Science. 284, $167-170$
Kosslyn, S. M., Thompson, W. L., \& Alpert, N. M. (1997). Neural systems shared by visual imagery and visual perception: A positron emission tomography study. Neuroimage. 6 , 320-334.

Kosslyn, S. M., Thompson, W. L., Kim, I. J., \& Alpert, N. M. (1995). Topographical representations of mental images in primary visual cortex. Nature. 378, 496-498.

Kosslyn, S. M., Thompson, W. L., Kim, I. J., Alpert, N. M., \& Rauch, S. L. (1996). Individual differences in cerebral blood flow in area 17 predict the time to evaluate visualized letters. Journal of Cognitive Neuroscience, 8, 78-82.

Le Bihan, D., Turner, R., Zeffiro, T. A., Cuénod, C. A., Jezzard, P., \& Bonnerot, V. (1993). Activation of primary visual cortex during visual recall: A magnetic resonance imaging study. Proceedings of the National Academv of Sciences. U.S.A. 90, 11802-11805.

Martinez, A., Anllo-Vento, L., Sereno, M. I., Frank, R., Buxton, R. B., Dubowitz, D. J., Wong, E. C., Hinrichs, H., Heinze, H. J., \& Hillyard, S. A. (1999). Involvement of striate and extrastriate visual cortical areas in spatial attention. Nature Neuroscience. 2, 364-369.

Mellet, E., Petit, L., Mazoyer, B., Denis, M., \& Tzourio, N. (1998). Reopening the imagery debate: Lessons from functional anatomy. Neuroimage, 8, 129-139.

Mellet, E., Tzourio, N., Denis, M., \& Mazoyer, B. (1998). Cortical anatomy of mental imagery of concrete nouns based on their dictionary definition. NeuroReport. 9, 803-808.

Mellet, E., Tzourio-Mazoyer, N., Bricogne, S., Mazoyer, B., Kosslyn, S. M., \& Denis, M. (2000). Functional anatomy of high-resolution visual mental imagery. Journal of Cognitive Neuroscience, 12, 98-109.

Pallier, C., Dupoux, E., \& Jeannin, X. (1997). Expe: An expandable programming language for psychological experiments. Behavior Research Methods, Instruments and Computers, 29, 322-327.

Richter, W., Ugurbil, K., Georgopoulous, A., \& Kim, S. G. (1997). Time-resolved fMRI of mental rotation. NeuroReport, 8, 3697-3702.

Roland, P. E., \& Gulyas, B. (1994). Visual imagery and visual representation. Trends in Neurosciences, 17, 281-287.

Roland, P. E., \& Gulyas, B. (1995). Visual memory, visual imagery and visual recognition of large field patterns in human brain: Functional anatomy by positron emission tomography. Cerebral Cortex, 5, 79-93.

Sakai, K., \& Miyashita, Y. (1994). Visual imagery: An interaction between memory retrieval and focal attention. Trends in Neurosciences. 17, 287-289.

Somers, D. C., Dale, A. M., Seiffert, A. E., \& Tootel, R. B. H. (1999). Functional MRI reveals spatially specific attentional modulation in human primary visual cortex. Proceedings of the National Academv of Sciences. U.S.A. 96, 1663-1668.

Talairach, J., \& Tournoux, P. (1988). Co-planar stereotaxic atlas of the buman brain. New York: Thieme.

Thompson, W. L., \& Kosslyn, S. M. (2000). Neural systems activated during visual mental imagery: A review and metaanalyses. In A. W. Toga \& J. C. Mazziotta (Eds.), Brain Mapping II: The Systems. San Diego: Academic Press.

Van de Moortele, P. F., Le Clec'h, G., Dehaene, S., \& Le Bihan, D. (1998). Improving auditory comprehension in event-related fMRI: Insertion of silent intervals in multi-slice EPI. Neuroimage, 7, S554.

Woodruff, P. W. R., Benson, R. R., Bandettini, P. A., Kwong, K. K., Howard, R. J., Talavage, T., Belliveau, J., \& Rosen, B. (1996). Modulation of auditory and visual cortex by selective attention is modality-dependent. NeuroReport. 7, 1909-1913. 


\section{This article has been cited by:}

1. Y. Masuda, S. O. Dumoulin, S. Nakadomari, B. A. Wandell. 2008. V1 Projection Zone Signals in Human Macular Degeneration Depend on Task, not Stimulus. Cerebral Cortex 18:11, 2483-2493. [CrossRef]

2. FranDois G. Meyer, Xilin Shen. 2008. <! [CDATA[Classification of fMRI Time Series in a Low-Dimensional Subspace With a Spatial Prior]]>. IEEE Transactions on Medical Imaging 27:1, 87. [CrossRef]

3. Huaien Luo, S. Puthusserypady. 2007. Estimation of the Hemodynamic Response of fMRI Data Using RBF Neural Network. IEEE Transactions on Biomedical Engineering 54:8, 1371-1381. [CrossRef]

4. M. Boly, E. Balteau, C. Schnakers, C. Degueldre, G. Moonen, A. Luxen, C. Phillips, P. Peigneux, P. Maquet, S. Laureys. 2007. Baseline brain activity fluctuations predict somatosensory perception in humans. Proceedings of the National Academy of Sciences 104:29, 12187-12192. [CrossRef]

5. ARDESHEER TALATI. 2005. VISUAL AND TACTILE GUIDANCE OF DEXTEROUS MANIPULATION TASKS: AN fMRI STUDY. Perceptual and Motor Skills 101:5, 317. [CrossRef]

6. David J. Kavanagh, Jackie Andrade, Jon May. 2005. Imaginary Relish and Exquisite Torture: The Elaborated Intrusion Theory of Desire. Psychological Review 112:2, 446-467. [CrossRef]

7. Catherine L. Reed, Shy Shoham, Eric Halgren. 2004. Neural substrates of tactile object recognition: An fMRI study. Human Brain Mapping 21:4, 236-246. [CrossRef]

8. Heledd C. Hart, Alan R. Palmer, Deborah A. Hall. 2004. Different areas of human non-primary auditory cortex are activated by sounds with spatial and nonspatial properties. Human Brain Mapping 21:3, 178-190. [CrossRef]

9. Miranda Occhionero. 2004. Mental Processes and the Brain During Dreams. Dreaming 14:1, 54-64. [CrossRef]

10. Stephen M. Kosslyn, William L. Thompson. 2003. When is early visual cortex activated during visual mental imagery?. Psychological Bulletin 129:5, 723-746. [CrossRef]

11. Ela I. Olivares , Jaime Iglesias , Socorro Rodríguez-Holguín . 2003. Long-Latency ERPs and Recognition of Facial IdentityLong-Latency ERPs and Recognition of Facial Identity. Journal of Cognitive Neuroscience 15:1, 136-151. [Abstract] [PDF] [PDF Plus]

12. Michael B. Miller, John Darrell Van Horn, George L. Wolford, Todd C. Handy, Monica Valsangkar-Smyth, Souheil Inati, Scott Grafton, Michael S. Gazzaniga. 2002. Extensive Individual Differences in Brain Activations Associated with Episodic Retrieval are Reliable Over TimeExtensive Individual Differences in Brain Activations Associated with Episodic Retrieval are Reliable Over Time. Journal of Cognitive Neuroscience 14:8, 1200-1214. [Abstract] [PDF] [PDF Plus]

13. A. Mazard , B. Mazoyer , O. Etard , N. Tzourio-Mazoyer , S.M. Kosslyn , E. Mellet . 2002. Impact of fMRI Acoustic Noise on the Functional Anatomy of Visual Mental ImageryImpact of fMRI Acoustic Noise on the Functional Anatomy of Visual Mental Imagery. Journal of Cognitive Neuroscience 14:2, 172-186. [Abstract] [PDF] [PDF Plus]

14. 2001. Current Awareness. NMR in Biomedicine 14:3, 217-222. [CrossRef]

15. Stephen M. Kosslyn, Giorgio Ganis, William L. ThompsonMental Imagery . [CrossRef] 